\title{
Independent and sensitive gait parameters for objective evaluation in knee and hip osteoarthritis using wearable sensors
}

\author{
Ramon J. Boekesteijn ${ }^{1,2^{*}}$, José M. H. Smolders ${ }^{3}$, Vincent J. J. F. Busch³, Alexander C. H. Geurts² and
} Katrijn Smulders ${ }^{1}$

\begin{abstract}
Background: Although it is well-established that osteoarthritis $(\mathrm{OA})$ impairs daily-life gait, objective gait assessments are not part of routine clinical evaluation. Wearable inertial sensors provide an easily accessible and fast way to routinely evaluate gait quality in clinical settings. However, during these assessments, more complex and meaningful aspects of daily-life gait, including turning, dual-task performance, and upper body motion, are often overlooked. The aim of this study was therefore to investigate turning, dual-task performance, and upper body motion in individuals with knee or hip OA in addition to more commonly assessed spatiotemporal gait parameters using wearable sensors.

Methods: Gait was compared between individuals with unilateral knee $(n=25)$ or hip OA $(n=26)$ scheduled for joint replacement, and healthy controls $(n=27)$. For $2 \mathrm{~min}$, participants walked back and forth along a 6-m trajectory making $180^{\circ}$ turns, with and without a secondary cognitive task. Gait parameters were collected using 4 inertial measurement units on the feet and trunk. To test if dual-task gait, turning, and upper body motion had added value above spatiotemporal parameters, a factor analysis was conducted. Effect sizes were computed as standardized mean difference between OA groups and healthy controls to identify parameters from these gait domains that were sensitive to knee or hip OA.

Results: Four independent domains of gait were obtained: speed-spatial, speed-temporal, dual-task cost, and upper body motion. Turning parameters constituted a gait domain together with cadence. From the domains that were obtained, stride length (speed-spatial) and cadence (speed-temporal) had the strongest effect sizes for both knee and hip OA. Upper body motion (lumbar sagittal range of motion), showed a strong effect size when comparing hip OA with healthy controls. Parameters reflecting dual-task cost were not sensitive to knee or hip OA.

(Continued on next page)
\end{abstract}

\footnotetext{
* Correspondence: r.boekesteijn@maartenskliniek.nl

'Department of Research, Sint Maartenskliniek, Hengstdal 3, 6574 NA Ubbergen, Nijmegen, The Netherlands

${ }^{2}$ Department of Rehabilitation, Donders Institute for Brain, Cognition and Behaviour, Radboud University Medical Center, Nijmegen, The Netherlands Full list of author information is available at the end of the article
}

(c) The Author(s). 2021 Open Access This article is licensed under a Creative Commons Attribution 4.0 International License, which permits use, sharing, adaptation, distribution and reproduction in any medium or format, as long as you give appropriate credit to the original author(s) and the source, provide a link to the Creative Commons licence, and indicate if changes were made. The images or other third party material in this article are included in the article's Creative Commons licence, unless indicated otherwise in a credit line to the material. If material is not included in the article's Creative Commons licence and your intended use is not permitted by statutory regulation or exceeds the permitted use, you will need to obtain permission directly from the copyright holder. To view a copy of this licence, visit http://creativecommons.org/licenses/by/4.0/ The Creative Commons Public Domain Dedication waiver (http://creativecommons.org/publicdomain/zero/1.0/) applies to the data made available in this article, unless otherwise stated in a credit line to the data. 
(Continued from previous page)

Conclusions: Besides more commonly reported spatiotemporal parameters, only upper body motion provided nonredundant and sensitive parameters representing gait adaptations in individuals with hip OA. Turning parameters were sensitive to knee and hip OA, but were not independent from speed-related gait parameters. Dual-task parameters had limited additional value for evaluating gait in knee and hip $\mathrm{OA}$, although dual-task cost constituted a separate gait domain. Future steps should include testing responsiveness of these gait domains to interventions aiming to improve mobility.

Keywords: Knee osteoarthritis, Hip osteoarthritis, Inertial measurement units, Gait analysis, Dual-task

\section{Background}

It is well-recognized that osteoarthritis (OA) of the knee or hip impairs gait [1-4]. Indeed, individuals with knee or hip OA walk less during daily life and their quality of gait is compromised [5]. Yet, objective gait assessments are not part of routine clinical evaluation, and gait difficulties in OA are insufficiently captured by patientreported outcome measures [6-8]. In part, this may be due to limited time available during clinical visits, considering that gait analysis is traditionally conducted in a gait laboratory, making it time consuming and not easily accessible. Recent advances in inertial sensor technology have opened up new avenues to quickly and objectively assess gait quality in a clinical setting.

Small inertial measurement units (IMUs) can be used to quickly and accurately obtain gait parameters without being restricted to a fixed (laboratory) environment [9, 10]. Moreover, compared to gait analysis in a lab, substantially more strides can be collected in a shorter period of time. On the downside, an important issue of gait assessment with IMUs is that it typically results in a large number of outcome parameters, with numerous correlated parameters. For example, many gait parameters share covariance with gait speed [11-15]. Hence, for clinical implementation, it is important to identify gait parameters from independent gait domains that best describe the gait adaptations in individuals with knee and hip OA compared to healthy controls.

So far, ambulatory gait assessments in individuals with knee and hip OA have mostly been limited to simple, straight-ahead walking paradigms [16]. Parameters reflecting more complex and relevant aspects of gait, including dual-task gait, turning, and compensatory trunk motion are less frequently reported in studies using IMUs. Turning and dual-task performance have been shown to be important aspects of daily life ambulation in elderly populations and can easily be assessed using wearable sensors [17-20]. Turning is a common cause of falling in community dwelling elderly, and may be more sensitive to sensorimotor impairments than straight-ahead gait [19, 21]. Dual-task performance, on the other hand, reflects the amount of attentional resources allocated to gait [22]. In order to compensate for gait difficulties caused by OA, a strategy could be to allocate more attention to gait. The extent to which a secondary cognitive task affects gait performance (i.e. dual-task cost (DTC)) may therefore be larger in individuals with OA. A recent scoping review indicated that DTC was not different between individuals with knee OA and healthy controls during quiet standing and forward induced falls [23]. However, DTC during gait has not yet been compared between those groups. A third gap in literature regarding wearable sensors and $\mathrm{OA}$ is the lack of attention for upper body movement. Upper body motion is important for maintaining stability, but may also be indicative of compensatory gait changes that reflect OA-related pain or disability [24-26].

The aim of this study was therefore to investigate turning, dual-task performance, and upper body motion in addition to spatiotemporal gait parameters in individuals with knee or hip OA, taking shared covariance between gait parameters into account. More specifically, we aim to test if 1) turning, dual-task gait, and upper body motion constitute independent domains of gait in our sample, and 2) gait parameters in these gait domains can discriminate individuals with knee or hip OA from healthy controls. Together, these findings may contribute to a better understanding of the multidimensional aspects of gait, and how this is affected in knee and hip OA.

\section{Methods \\ Participants}

In this cross-sectional, comparative study 78 participants were included. The total study population comprised three groups: individuals with unilateral knee OA $(n=$ 25), unilateral hip OA $(n=26)$, and healthy controls $(n=27)$. Samples were derived from a longitudinal study investigating gait before and after total knee and hip arthroplasty that was powered for the comparison of spatiotemporal gait characteristics between individuals 1 year after total knee or hip arthroplasty and healthy controls. Individuals with $\mathrm{OA}$ were recruited at the Sint Maartenskliniek and were included if they had both radiological and symptomatic OA and were listed for joint replacement surgery. Participants had to be able to walk for more than 2 min without the use of any assistive device. Exclusion criteria were: 1) expectancy of joint 
replacement within a year, or symptomatic OA, in another weight-bearing joint than the joint scheduled for surgery, 2) BMI $>40 \mathrm{~kg} / \mathrm{m}^{2}$, and 3) any other musculoskeletal or neurological impairment interfering with gait or balance. Healthy controls were recruited from the community and did not have a clinical diagnosis of knee or hip OA, nor did they have any pain in the lower extremities. Healthy controls were recruited in the same age range as individuals with OA. Exclusion criteria for healthy controls were the same as for individuals with knee and hip OA. Informed consent was obtained from all participants prior to testing. Ethical approval was obtained from the CMO Arnhem/Nijmegen (2018-4452). All study methods were carried out in accordance with the Declaration of Helsinki.

\section{Demographic and clinical assessment}

Evidence for radiological OA was provided by the Kellgren and Lawrence $(\mathrm{KL})$ score as assessed by experienced orthopedic surgeons [27]. Anthropometric characteristics were obtained during the pre-operative screening visit and were summarized as mass, length, and BMI. For individuals with knee and hip OA, self-reported functioning was assessed using the Knee Injury and Osteoarthritis Outcomes Score (KOOS) or Hip Disability Osteoarthritis Outcome Score (HOOS) [28, 29]. All items were scored on a zero to four Likert scale. For the five subscales, total scores were transformed to a 0-100 scale, with 100 representing best function.

\section{Gait assessment}

Gait parameters were collected on the same day as the pre-operative screening visit, which took place approximately 1 to 2 months prior to surgery. Four IMUs (Opal V2, APDM Inc., Portland, OR) were used to obtain segment accelerations and angular velocities (sample frequency $=128 \mathrm{~Hz}$ ). Sensors were attached via elastic straps to the dorsum of both feet, the waist (sacrolumbar level), and the sternum (Fig. 1) according to the standardized sensor placement of MobilityLab. Participants walked wearing flat shoes at a self-selected comfortable speed. For a duration of 2 min, participants walked back and forth along a 6-m trajectory making $180^{\circ}$ turns (Fig. $1)$. Two 2-min trials were collected, with and without a secondary cognitive task. The cognitive task consisted of an alternating alphabet task, citing every other letter of the alphabet. Single-task walking was always performed before the dual-task condition. Responses to the cognitive task were recorded by the assessor. Accuracy on the cognitive task was summarized as correct responses (percentage of total responses). DTC was computed as the percentual change of dual-task performance relative to the single-task for the following parameters: gait speed, cadence, stride length, stride time variability, and turn duration.

\section{Data analysis}

Gait parameters were extracted from the raw IMU signals using the commercially available and validated Mobility Lab v2.0 software package [30]. Mobility Lab uses a state space model with causal Kalman filter along with zero velocity updates for optimal orientation estimation. Range of motion metrics were described for both the lumbar and trunk sensors using the gyroscope signals. As such, these measures are representative of the rotation of the sensors, which is caused by the movement of the underlying segments. For parameters where side was relevant (i.e. foot elevation at midswing, lateral step variability, circumduction, foot strike angle, toe off angle, and stance duration), we analyzed the affected leg in

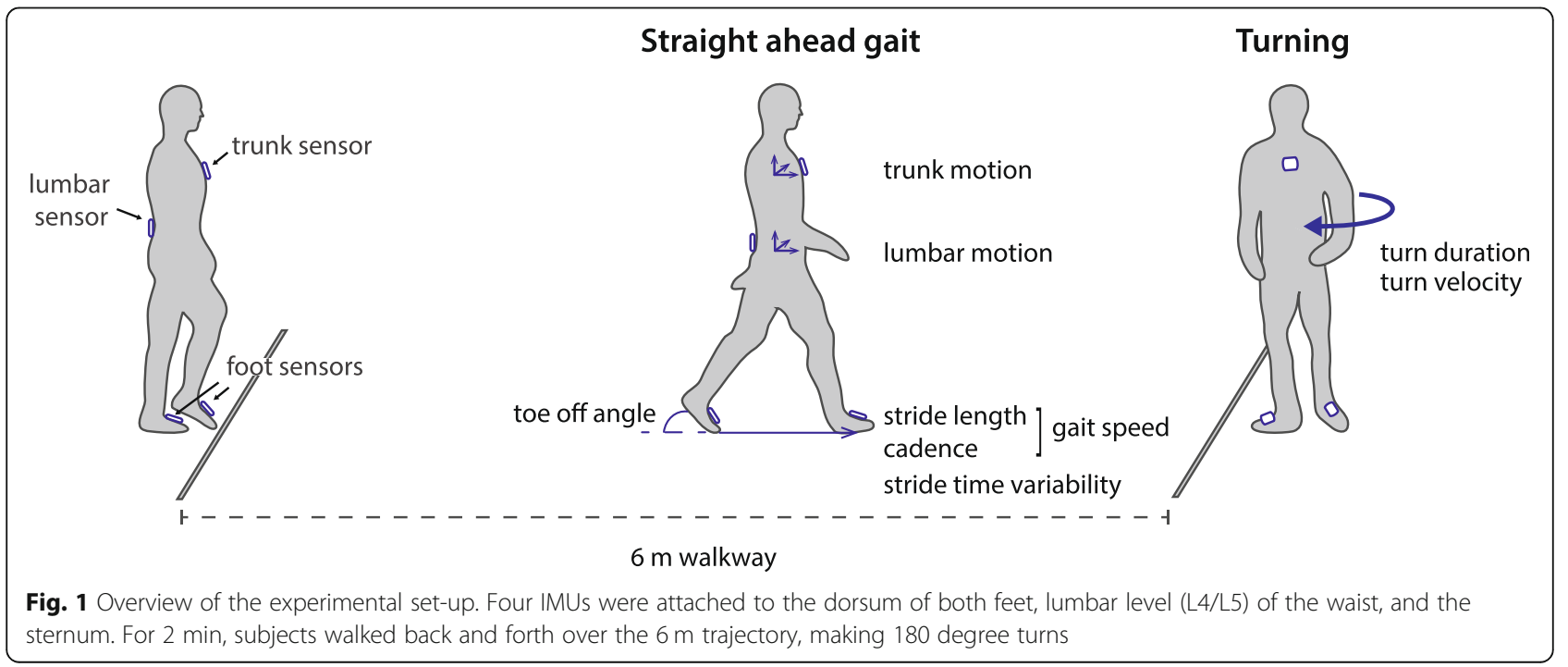


individuals with knee or hip OA, whereas for healthy controls the average value from the left and right leg was taken. Gait parameters were initially selected based on reliability, theoretical considerations, and completeness $(<20 \%$ missing values). Based on the reliability criterium, we excluded stance and swing duration as percentage of gait cycle [31]. With regard to theoretical considerations, the following decisions were made: 1) in case gait parameters reflected the same outcome (e.g. gait cycle duration and cadence) only one parameter was kept for further analysis, 2) asymmetry parameters were restricted to meaningful parameters (i.e. stride length cannot be asymmetric when walking over a straight path) [32]. DTC of gait parameters that are ratios (i.e. asymmetry values) were not included in order to prevent inflated values, except for stride time variability, due to the substantial number of other studies evaluating this parameter in the context of fall risk [33]. This resulted in twenty-five gait parameters entered into factor analysis to identify correlated outcomes.

Exploratory factor analysis was used to identify independent gait domains explaining the variance in gait performance. Adequacy of the dataset for factor analysis was tested using Barlett's test of sphericity and the Kaiser-Meyer-Olkin (KMO) test. In case individual KMO values were lower than 0.5 , variables were removed from the analysis [34]. The number of factors to be retained for further analysis was determined using the Kaiser criterium (eigenvalue >1.0) [35]. Subsequently, factor analysis with varimax rotation was performed to obtain orthogonal factor scores. Within a factor, gait parameters were considered relevant when they met a minimum factor loading of 0.5 .

For each relevant gait parameter in the obtained factor, effect sizes were computed as standardized mean differences (SMD) for the comparison between the OA groups and healthy controls (knee OA vs healthy controls and hip OA vs healthy controls). The gait parameter with the highest factor loading in combination with an effect size larger than 0.5 was considered nonredundant and sensitive to either knee or hip OA. For these gait parameters, individual datapoints and means with 95\% confidence intervals (CI) were constructed using estimation graphs to assess between-group differences [36].

For demographic and clinical parameters, main group effects (3 levels: knee OA, hip OA, healthy controls) were tested using a one-way ANOVA or non-parametric equivalent when assumptions for parametric testing were not met. In case of a significant main effect, a posthoc comparison was conducted using independent samples Student's t-test or the non-parametric equivalent. Data was considered statistically significant at an alpha level of 0.05 , which was adjusted for multiple comparisons $(n=9)$ for the gait parameters. This resulted in a Bonferroni adjusted alpha level of 0.0056 . Data analysis was performed using STATA and customwritten Python scripts incorporating the DABEST library [37].

\section{Results \\ Participant characteristics}

Age, sex, and height did not differ between OA groups and healthy controls (Table 1). Individuals with knee OA had - on average - a $9 \mathrm{~kg}$ (95\% CI: $2-16$; $p=0.014)$ higher mass compared to healthy controls. This difference was $12 \mathrm{~kg}$ (95\% CI: $3-20 ; p=0.007)$ between individuals with hip $\mathrm{OA}$ and healthy controls. For individuals with knee $\mathrm{OA}$, this translated into a $2.8 \mathrm{~kg} /$ $\mathrm{m}^{2}$ (95\% CI: 0.9-4.7; $p=0.005$ ) higher mean BMI compared to the control group, whereas the mean BMI was $2.4 \mathrm{~kg} / \mathrm{m}^{2}$ (95\% CI: 0.1-4.7; $p=0.043$ ) higher in individuals with hip OA. Severity of radiographic OA was moderate to severe $\mathrm{OA}(\mathrm{KL}=3$ or 4$)$ in both groups. Furthermore, accuracy on the secondary cognitive task was comparable between individuals with knee (mean: $84 \%$ ) or hip OA (mean: $87 \%$ ) and healthy controls (mean: 89\%). KOOS and HOOS scores indicated presence of pain, disability, and limited quality of life in individuals with knee and hip OA (Table 1). Gait parameters were based on 32 valid strides (95\% CI: 29-36) in individuals with knee OA, 34 valid strides (95\% CI: 31-37) in individuals with hip OA, and 30 valid strides $(95 \% \mathrm{CI}$ : 27-34) in healthy participants.

\section{Exploratory factor analysis}

Twenty-five gait parameters were entered into the factor analysis (Fig. 2). Based on individual KMO values, the following variables were removed from further analysis: DTC of stride length, trunk transverse range of motion (RoM), lateral step variability, toe-out angle, and foot elevation at midswing. Factor analysis of the remaining twenty parameters yielded four orthogonal factors accounting for $87.8 \%$ of the total variance in gait performance (Table 2). The factors were described as speedspatial, speed-temporal, dual-task cost, and upper body motion. Gait speed had a cross-loading on the factors speed-spatial (0.759) and speed-temporal (0.579). Turning parameters loaded on the factor speed-temporal. In the upper body motion domain, factor loadings of the parameters were relatively low, ranging between 0.53 and 0.61 .

\section{Selection of gait parameters based on effect size}

SMDs for the comparison between OA groups and healthy participants are visualized for all gait parameters in Fig. 3. Based on the criterium for effect size, the following gait parameters were selected to represent the 
Table 1 Demographic and clinical characteristics of all three subject groups

\begin{tabular}{|c|c|c|c|c|c|}
\hline Parameter & $\begin{array}{l}\text { Controls } \\
(n=27)\end{array}$ & $\begin{array}{l}\text { Knee OA } \\
(n=25)\end{array}$ & $\begin{array}{l}\text { Hip OA } \\
(n=26)\end{array}$ & $\begin{array}{l}\text { ANOVA main } \\
\text { group effect }\end{array}$ & Post-hoc comparisons \\
\hline Age (y) & $66[63-68]$ & $64[61-67]$ & $64[61-66]$ & $F(2,75)=0.67, p=0.514$ & - \\
\hline Sex (M:F) & $13: 14$ & $12: 13$ & $17: 9$ & $\begin{array}{l}X^{2}(2, N=78)=2.09 \\
p=0.352\end{array}$ & - \\
\hline Height (m) & $1.72[1.68-1.75]$ & $1.72[1.68-1.77]$ & $1.76[1.73-1.80]$ & $F(2,75)=1.72, p=0.185$ & - \\
\hline Mass (kg) & $76[72-80]$ & 84 [79-90] & 88 [80-95] & $F(2,75)=4.51, \boldsymbol{p}=\mathbf{0 . 0 1 4}$ & $\begin{array}{l}\text { Knee OA vs HC: } \text { mean diff }=\mathbf{9}[\mathbf{2}-16] \\
p=0.014 \\
\text { Hip OA vs HC: } \text { mean diff }=\mathbf{1 2}[\mathbf{3}-\mathbf{2 0}] \\
p=0.007\end{array}$ \\
\hline BMI $\left(\mathrm{kg} / \mathrm{m}^{2}\right)$ & $25.7[24.6-26.8]$ & $28.5[26.9-30.1]$ & $28.1[26.0-30.1]$ & $F(2,75)=3.52, \boldsymbol{p}=\mathbf{0 . 0 3 5}$ & $\begin{array}{l}\text { Knee OA vs HC: } \text { mean diff }=\mathbf{2 . 8}[\mathbf{0 . 9 - 4 . 7}] \\
p=0.005 \\
\text { Hip OA vs HC: } \text { mean diff }=\mathbf{2 . 4}[\mathbf{0 . 1}-\mathbf{4 . 7}] \\
p=0.043\end{array}$ \\
\hline KL score (I:II:II:IV) & - & $0: 0: 8: 17$ & $0: 0: 7: 19$ & - & - \\
\hline $\begin{array}{l}\text { DT scores } \\
\text { (\% correct) }\end{array}$ & 89 [86-92] & 84 [79-89] & 87 [84-91] & $F(2,75)=1.56, p=0.217$ & - \\
\hline Self-reported outcomes & & KOOS & HOOS & & \\
\hline 1) Symptoms & - & $50.9[42.5-59.3]$ & $41.4[33.6-49.2]$ & - & - \\
\hline 2) Pain & - & $41.7[33.8-49.5]$ & $39.6[34.4-44.8]$ & - & - \\
\hline 3) Activities of daily life & - & 52.9 [44.9-60.9] & $39.7[33.7-45.6]$ & - & - \\
\hline 4) Sport/ Recreation & - & $15.6[7.9-23.3]$ & $15.1[10.5-19.8]$ & - & - \\
\hline 5) Quality of life & - & 26.0 [20.4-31.6] & $23.6[17.8-29.3]$ & - & - \\
\hline
\end{tabular}

Data are presented as mean [95\% Cl]. Significant differences are bold

OA osteoarthritis, KL Kellgren and Lawrence, BMI body mass index, DT dual-task, HC healthy controls, HOOS hip disability and osteoarthritis outcome score, KOOS knee injury and osteoarthritis outcome score

corresponding factors: stride length (speed-spatial), cadence (speed-temporal), and lumbar sagittal RoM (upper body motion). Although the factor DTC explained $20.7 \%$ of the total variance in gait performance, none of the gait parameters within this factor showed an effect size larger than 0.5 (Fig. 3). Gait speed showed the largest effect size for both the comparison between knee OA and controls $(\mathrm{SMD}=1.59)$ and hip $\mathrm{OA}$ and controls $(\mathrm{SMD}=$ 1.70). However, due to cross-loadings on factors speedspatial and speed-temporal, gait speed was not prioritized over stride length and cadence. In addition, many of the gait parameters from the factor speed-spatial and speed-temporal showed large effect sizes (SMD > 0.8) for both group comparisons.

\section{Between group comparisons of non-redundant gait parameters}

Between-group differences of the selected gait parameters were visualized using estimation plots (Fig. 4). Both individuals with knee and hip OA walked with a lower cadence and with shorter steps. More specifically, compared to healthy controls stride length was $0.17 \mathrm{~m}$ (95\% CI: 0.09-0.26, $p<0.001)$ lower in individuals with knee OA and $0.20 \mathrm{~m}$ (95\% CI: $0.12-0.28, p<0.001)$ lower in hip OA. In addition, cadence was 10.8 steps/min (95\% CI: 6.3-15.4, $p<0.001)$ lower in individuals with knee OA and 9.8 steps/min (95\% CI: 5.2-14.4, $p<0.001$ ) lower in individuals with hip OA. Lumbar RoM in the sagittal plane was 2.7 degrees (95\% CI: 1.7-4.4, $p<$ 0.001 ) higher for individuals with hip OA compared to controls, whereas no differences were found between knee OA individuals and healthy controls (mean difference $=0.5$ degrees, 95\% CI: $-0.33-1.59, p=0.260$ ).

\section{Discussion}

The aim of the present study was to investigate turning, dual-task performance, and upper body motion in addition to spatiotemporal gait parameters in individuals with knee or hip OA. To avoid redundancy of gait parameters, we conducted a factor analysis. Four independent gait domains were identified: speed-spatial, speedtemporal, dual-task cost, and upper body motion. Turning did not constitute its own domain but was related to speed-temporal. Three domains held parameters sensitive to knee or hip OA: speed-spatial (stride length), speed-temporal (cadence), and upper body motion (lumbar sagittal RoM). Dual-task cost was not sensitive to knee or hip OA.

Factor analysis effectively reduced the dimensionality of our dataset from twenty-five gait parameters to four independent domains of gait, including domains related to dual-task gait and compensatory trunk motion. Turning, however, was part of a factor together with cadence. The factors explaining most of the variance in our 


\section{Pre-selected gait parameters $(\mathbf{n}=\mathbf{2 5})$}

1. Cadence (steps/min)

2. Foot elevation at midswing $(\mathrm{cm})$

3. Lateral step variability $(\mathrm{cm})$

4. Circumduction $(\mathrm{cm})$

5. Foot strike angle (deg)

6. Toe off angle (deg)

7. Stance duration $(\%)$

8. Toe out angle (deg)

9. Stride time CV (\%)

10. Gait speed $(\mathrm{m} / \mathrm{s})$

11. Step duration Asymmetry (\%)

12. Stride length $(\mathrm{m})$

13. Lumbar coronal RoM (deg)
14. Lumbar sagittal RoM (deg)

15. Lumbar transverse RoM (deg)

16. Trunk coronal RoM (deg)

17. Trunk sagittal RoM (deg)

18. Trunk transverse RoM (deg)

19. Turn duration (s)

20. Turns - peak velocity (deg/s)

21. DTC cadence $(\%)$

22. DTC stride time CV (\%)

23. DTC gait speed (\%)

24. DTC stride length (\%)

25. DTC turn duration (\%)

\begin{tabular}{|l|lc|}
\hline & \multicolumn{3}{|c|}{ Removed based on individual KMO values (n=5) } \\
\cline { 2 - 3 } & 1. DTC stride length & 0.287 \\
2. Trunk transverse RoM & 0.327 \\
3. Lateral step variability & 0.453 \\
4. Toe-out angle & 0.435 \\
5. Foot elevation at midswing & 0.479 \\
\hline
\end{tabular}

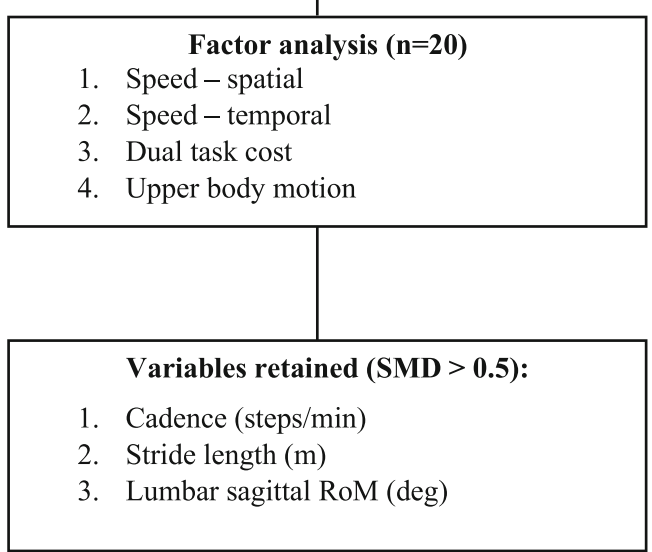

Fig. 2 Flowchart describing the selection process of gait parameters. Note: foot elevation at midswing = height of the foot sensor at mid-swing, lateral step variability = spatial deviation in the lateral direction of each foot compared to previous steps, circumduction = amount that the foot travels perpendicular to forward movement during the swing phase

sample, i.e. speed-spatial and speed-temporal, were both dependent on gait speed (Table 2). In the literature, these factors reflecting the spatial and temporal aspects of gait speed are consistently reported [38-42]. Other factors related to gait are variability [38, 39, 41, 42], asymmetry [39, 41, 42], postural control [39], and trunk motion [40]. Dual-task cost has not previously been evaluated in a factor analysis approach, but may contain unique information about gait that is informative of disease-specific compensations related to the reallocation of attentional resources. Importantly, dualtask cost and upper body motion are interesting domains as they were independent of gait speed, evidenced by the absence of a cross-loading of gait speed on these domains in our study. Dual-task cost and upper body motion may therefore provide promising gait parameters for clinical evaluation of gait, in addition to the more commonly used speed-related measures.

In our analysis steps, turning parameters were excluded in favor of the stronger factor loading that was obtained for cadence. However, effect sizes for turning were large when comparing both knee and hip OA with healthy participants (SMD $>0.9$, Fig. 3). In addition, factor loadings were not substantially lower compared to cadence. Taken together, we are unsure whether this factor represents a combination of gait and turning, or better reflects turning itself. Future research should therefore indicate as to what extent turning parameters 
Table 2 Item loadings obtained from the factor analysis $(n=4)$ with varimax rotation

\begin{tabular}{|c|c|c|c|c|}
\hline Gait parameters & Speed-spatial & Speed-temporal & Dual-task cost & Upper body motion \\
\hline Stride Length $(\mathrm{m})$ & 0.907 & 0.270 & 0.050 & -0.000 \\
\hline Gait Speed (m/s) & 0.759 & 0.579 & 0.170 & -0.062 \\
\hline Foot Strike Angle (deg) & 0.742 & 0.120 & -0.161 & 0.257 \\
\hline Toe Off Angle (deg) & 0.628 & 0.267 & 0.129 & -0.233 \\
\hline Stride Time CV (\%) & -0.596 & -0.260 & -0.077 & -0.051 \\
\hline Cadence (steps/min) & 0.203 & 0.830 & 0.284 & -0.163 \\
\hline Turns - Peak velocity (deg/s) & 0.420 & 0.745 & -0.090 & 0.102 \\
\hline Turn Duration (s) & -0.453 & -0.704 & 0.108 & 0.092 \\
\hline DTC Cadence (\%) & 0.067 & 0.010 & 0.935 & 0.047 \\
\hline DTC Gait Speed (\%) & 0.060 & 0.107 & 0.921 & 0.057 \\
\hline Lumbar Sagittal RoM (deg) & 0.113 & -0.159 & 0.028 & 0.611 \\
\hline Lumbar Transverse RoM (deg) & 0.029 & 0.134 & 0.131 & 0.562 \\
\hline Trunk Sagittal RoM (deg) & 0.008 & -0.221 & 0.111 & 0.543 \\
\hline Trunk Coronal RoM (deg) & -0.049 & -0.123 & -0.008 & 0.528 \\
\hline Explained variance (\%) & 30.0 & 22.5 & 20.7 & 14.6 \\
\hline
\end{tabular}

Barlett's test of sphericity confirmed absence of an identity matrix $\left(X^{2}(190)=1447.09, p<0.001\right)$. Suitability of the dataset was indicated by the Kaiser-Meyer-Olkin measure, which was 0.666 . Together the four factors explained $87.8 \%$ of the variance in our sample $C V$ coefficient of variation, DTC dual-task cost, RoM range of motion

are driven by cadence or gait speed, and how meaningful the unexplained variance is for evaluation of physical functioning in individuals with knee and hip OA.

To facilitate assessment of the between-group differences, we opted to select single gait parameters from the independent factor, to represent the respective factor. From the factors that we obtained, only dual-tasking parameters did not discriminate between knee or hip OA and healthy controls $(\mathrm{SMD}<0.5)$. This indicates that, compared to healthy controls, individuals with OA did not need more attentional resources for the motor task.
Thus, although gait was affected in OA, this was not compensated by more attentional resources.

Many of the gait parameters that showed large between-group effect sizes (Fig. 3) were grouped either under the speed-spatial or under the speed-temporal domain. This suggests that the two main components determining gait speed, stride length and cadence, are inherently linked with various gait adaptations prominent in individuals with knee and hip OA. As such, gait speed may also be considered as the final common pathway for various gait adaptations, and could be used as a

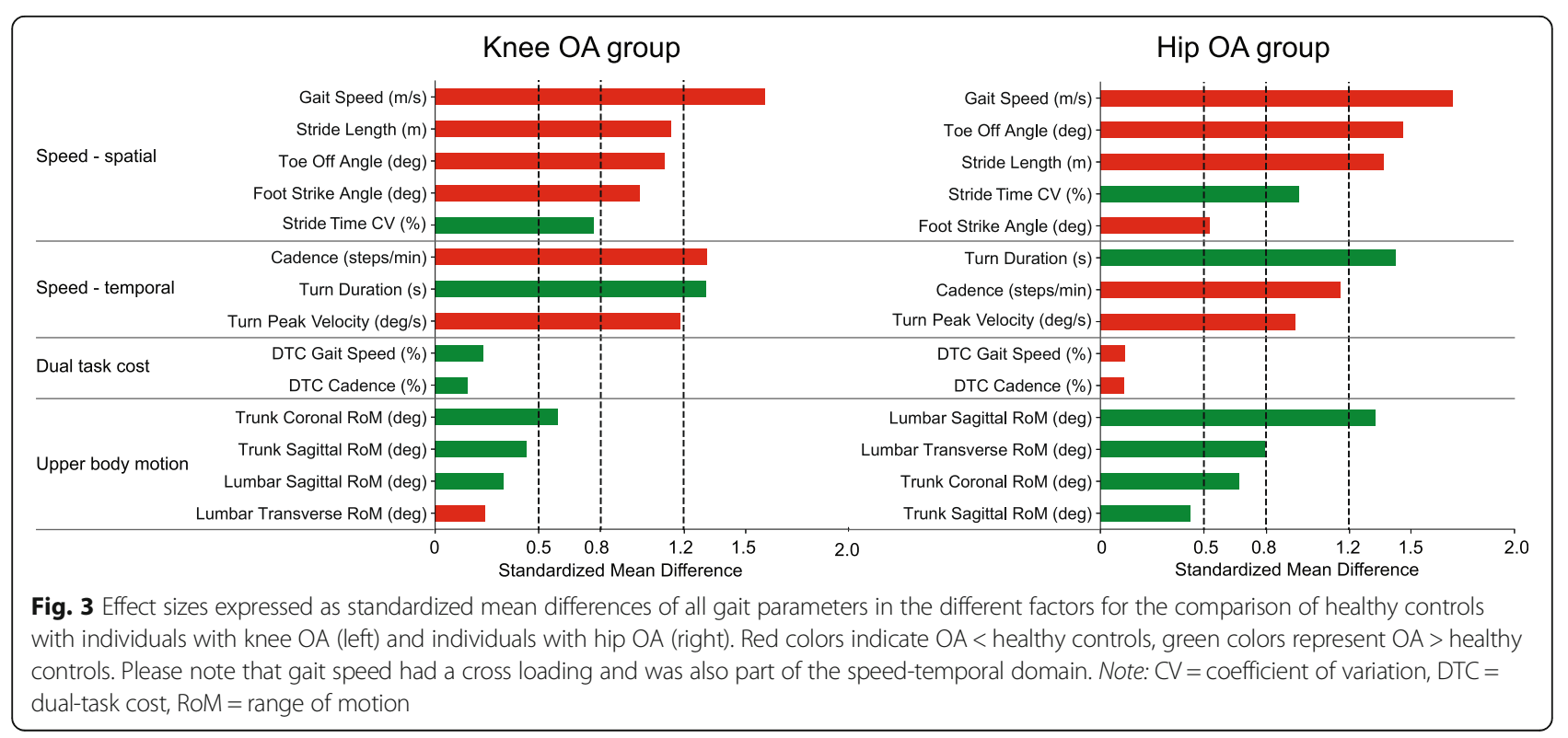



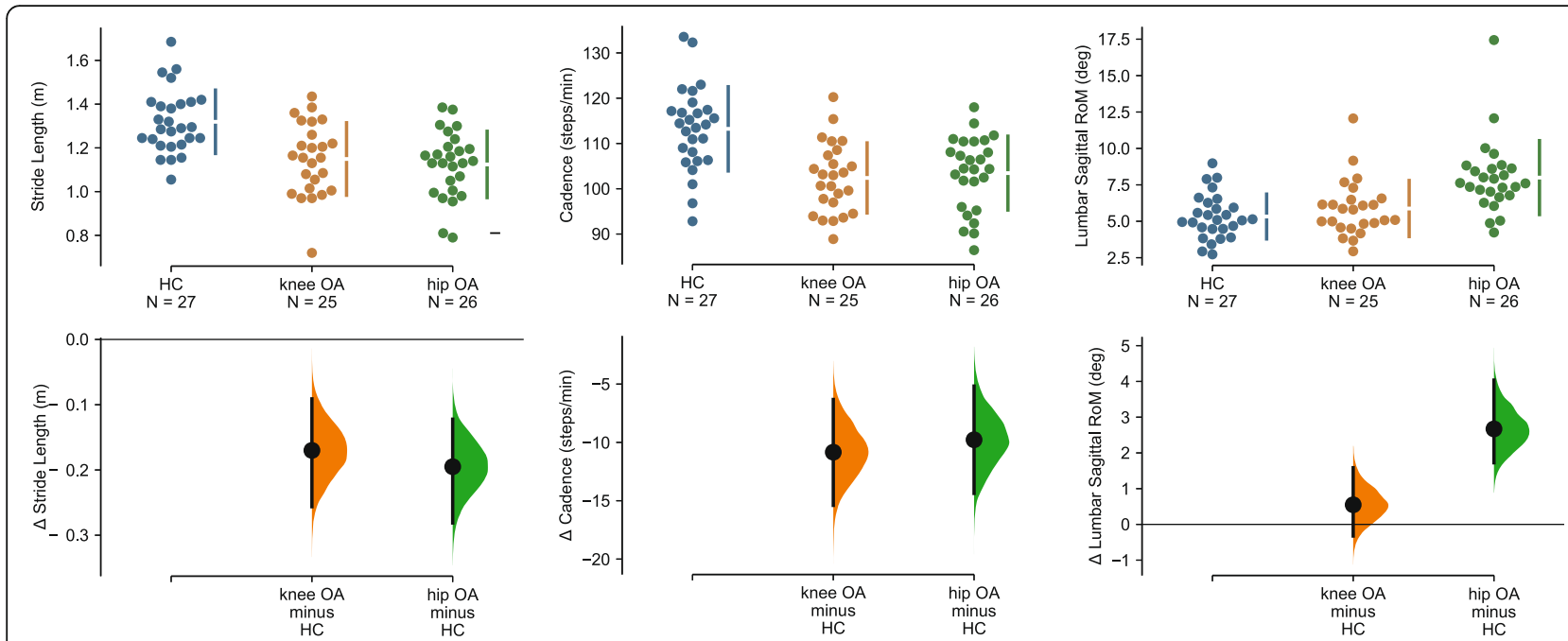

Fig. 4 Estimation plots of the mean group differences for stride length, cadence, and lumbar sagittal RoM. In the top panel, dots represent the individual datapoints and bars the mean ( \pm SD). In the bottom panel, the distribution of the mean difference $( \pm 95 \%$ Cl) for the comparison with healthy controls is visualized. In cases where zero is not in the $95 \% \mathrm{Cl}$ of the mean difference, as indicated by the black bars in the lower panels, data was statistically different at $p<0.05$

very general, but highly sensitive marker for functional status in individuals with OA. Next to this, our findings further stress the need to take gait speed differences into account when evaluating gait in individuals with OA. More specifically, for parameters that are correlated with gait speed, it may be more appropriate to assess them at a standardized, matched speed, as it may be difficult to separate effects of gait speed from the effects of OA itself [43]. Finally, these findings underline the importance of data reduction techniques when investigating gait using IMUs or motion capture systems, as statistical testing of all gait parameters would increase the probability of finding false positives.

That speed-related gait parameters have good discriminatory capacity in OA has been reported before. Two systematic reviews reported lower gait speed and stride length in individuals with knee and hip OA compared to healthy participants $[1,3]$. In studies employing IMUs, similar changes in stride length and cadence were found $[25,44]$. In absolute numbers, slight differences with our values can be discerned. Reasons for this may include the relatively short walking distance $(6 \mathrm{~m})$ in this study that was necessary to reliably assess turning, versus the longer distances $(\sim 20 \mathrm{~m})$ that are commonly used. Nevertheless, our findings corroborated previous findings about the discriminatory capacity of stride length and cadence.

In addition to spatiotemporal differences, individuals with hip OA walked with distinct upper body motion, which was most evident in the sagittal plane at the lumbar level. However, upper body motion is difficult to capture by just one parameter, as is illustrated by the relatively low factor loadings lying close together in this domain (Table 1). Altered trunk motion may point toward the use of compensatory strategies to unload the arthritic joint [45]. More specifically, increased pelvic RoM in the sagittal plane may enable more effective anteflexion of the lower limbs and may thereby, to a certain extent, preserve stride length [46]. In addition, anterior pelvic tilt combined with lateral trunk lean can reduce the lever arm between the hip joint center and center of mass [25]. We observed more lumbar sagittal RoM and more RoM of the trunk in the coronal plane in individuals with hip OA compared to healthy controls, in line with previous reports [25, 46]. Unfortunately, the exact reason for the use of these compensatory mechanisms remains speculative and may relate to pain, muscle weakness, or joint instability [47]. Future research should therefore investigate the importance of upper body motion in individuals with OA, to inform us about potential mechanisms underlying these gait adaptations.

With regard to the use of wearable sensors in clinical practice, our study showed that quick and easy gait assessments with wearable sensors are useful for evaluating gait impairments in individuals with knee and hip OA. In comparison to optical motion capture systems, wearable sensors are more feasible for large-scale use and could be utilized to routinely assess physical functioning. From all gait parameters, gait speed was found to be a very general but highly sensitive marker for mobility limitations, combining both the effects on stride 
length and cadence. Besides the basic spatiotemporal measures, trunk motion and turning appeared to be relevant for individuals with knee and hip OA. We therefore recommend to use sensor configurations that allow to look beyond these basic spatiotemporal parameters. In the future, wearable sensors should also be utilized to their fullest potential to enable remote monitoring at home, which would allow to more accurately capture the habitual gait patterns.

This study had several limitations that merit attention. First, we did not obtain factors representing gait asymmetry or variability, which may have been related to the low number of gait parameters related to those domains that were initially entered into factor analysis. We were therefore limited in our conclusions regarding the potential value of those measures for individuals with knee or hip OA. Second, five potentially valuable gait parameters were removed from further analysis due to sampling inadequacy (KMO value $<0.5$ ). Larger sample sizes are therefore required to identify the potential value of these parameters. Related to this, we did not include demographic or clinical variables in the factor analysis, as this could have affected the accuracy of factor analysis due to the relatively small sample size. Finally, including individuals with isolated, unilateral knee or hip OA was important for our study purposes, although the majority of the OA population have complaints in more than one joint [48]. We expect that widening the inclusion criteria would have resulted in larger differences of OA groups compared to healthy controls, but in less specificity for each OA group. In addition, it is important to note that individuals in this study had end-stage OA and were scheduled for joint replacement. Our results may thus not be representative of gait in individuals with less severe $\mathrm{OA}$.

\section{Conclusion}

In addition to commonly assessed spatiotemporal parameters, this study provided two other relevant domains of gait: dual-task cost and upper body motion. Although dual-task cost provided unique information about gait, our results did not suggest that individuals with knee or hip OA needed more attention for walking than healthy participants. Adaptations in upper body motion were more subtle than stride length and cadence, but may carry important information about compensatory strategies that are most distinctive for individuals with hip OA. Future steps should include evaluation of the responsiveness of these gait parameters to effects of interventions aiming to improve mobility, such as joint replacement surgery. Furthermore, longitudinal monitoring of individuals with knee and hip OA starting at earlier stages of the disease may inform us about the development of these gait adaptations and associated compensations over time.

\section{Supplementary Information}

The online version contains supplementary material available at https://doi. org/10.1186/s12891-021-04074-2.

\section{Additional file 1.}

\section{Abbreviations}

OA: Osteoarthritis; IMU: Inertial measurement unit; DTC: Dual-task cost; KL: Kellgren and Lawrence; KOOS: Knee injury and osteoarthritis outcome score; HOOS: Hip disability and osteoarthritis outcome score; KMO: KaiserMeyer-Olkin; SMD: Standardized mean difference; Cl: Confidence interval; RoM: Range of motion

\section{Acknowledgements}

We want to thank Jule Mevis, Eelco van Leent, and Roosmarijn Brenninkmeijer for their contributions to the data collection.

\section{Authors' contributions}

$J S, V B$, and KS designed the study and obtained funding. RB and KS collected the data. RB and KS analyzed and interpreted the data. JS and VB scored the radiological images. RB and KS prepared first draft version of the manuscript. AG and KS were responsible for supervision of the project. All authors read and approved the final version of the article.

\section{Funding}

The Innovation Fund of the Sint Maartenskliniek sponsored this study. The funders had no role in the design and conduct of the study.

\section{Availability of data and materials}

The dataset supporting the conclusions of this article is included within the article and its additional files.

\section{Ethics approval and consent to participate}

All experimental procedures were approved by the ethics committee $\mathrm{CMO}$ Arnhem/Nijmegen (2018-4452). Informed consent was obtained from all participants prior to testing.

\section{Consent for publication}

Not applicable.

\section{Competing interests}

The authors declare they have no competing interests.

\section{Author details}

${ }^{1}$ Department of Research, Sint Maartenskliniek, Hengstdal 3, 6574 NA Ubbergen, Nijmegen, The Netherlands. ${ }^{2}$ Department of Rehabilitation, Donders Institute for Brain, Cognition and Behaviour, Radboud University Medical Center, Nijmegen, The Netherlands. ${ }^{3}$ Department of Orthopedic Surgery, Sint Maartenskliniek, Nijmegen, The Netherlands.

Received: 2 November 2020 Accepted: 9 February 2021

Published online: 03 March 2021

\section{References}

1. Ornetti P, Maillefert J-F, Laroche D, Morisset C, Dougados M, Gossec L. Gait analysis as a quantifiable outcome measure in hip or knee osteoarthritis: A systematic review. Jt Bone Spine. 2010;77:421-5. https://doi.org/10.1016/j. jbspin.2009.12.009.

2. Mills K, Hunt MA, Ferber R. Biomechanical deviations during level walking associated with knee osteoarthritis: A systematic review and meta-analysis. Arthritis Care Res (Hoboken). 2013;65. https://doi.org/10.1002/acr.22015.

3. Constantinou M, Barrett R, Brown M, Mills P. Spatial-Temporal Gait Characteristics in Individuals With Hip Osteoarthritis: A Systematic Literature Review and Meta-analysis. J Orthop Sport Phys Ther. 2014;44:291-B7. https://doi.org/10.2519/jospt.2014.4634.

4. Winter CC, Brandes M, Müller C, Schubert T, Ringling M, Hillmann A, et al. Walking ability during daily life in patients with osteoarthritis of the knee or the hip and lumbar spinal stenosis: a cross sectional study. BMC Musculoskelet Disord. 2010;11:233. https://doi.org/10.1186/1471-24 74-11-233. 
5. Brandes M, Schomaker R, Möllenhoff G, Rosenbaum D. Quantity versus quality of gait and quality of life in patients with osteoarthritis. Gait Posture. 2008;28:74-9. https://doi.org/10.1016/j.gaitpost.2007.10.004.

6. Terwee $C B$, van der Slikke RMA, van Lummel RC, Benink RJ, Meijers WGH, de Vet HCW. Self-reported physical functioning was more influenced by pain than performance-based physical functioning in knee-osteoarthritis patients. J Clin Epidemiol. 2006;59:724-31. https://doi.org/10.1016/j.jclinepi.2005.11.01 9.

7. Fransen BL, Mathijssen NMC, Slot $\mathrm{K}$, de Esch NHH, Verburg H, Temmerman OPP, et al. Gait quality assessed by trunk accelerometry after total knee arthroplasty and its association with patient related outcome measures. Clin Biomech. 2019;70:192-6. https://doi.org/10.1016/j.clinbiomech.2019.10.007.

8. Bolink SAAN, Lenguerrand E, Brunton LR, Wylde V, Gooberman-Hill R, Heyligers IC, et al. Assessment of physical function following total hip arthroplasty: Inertial sensor based gait analysis is supplementary to patientreported outcome measures. Clin Biomech. 2016;32:171-9. https://doi.org/1 0.1016/j.clinbiomech.2015.11.014.

9. Tao W, Liu T, Zheng R, Feng H. Gait Analysis Using Wearable Sensors. Sensors. 2012;12:2255-83. https://doi.org/10.3390/s120202255.

10. Kobsar D, Charlton JM, Tse CTF, Esculier J-F, Graffos A, Krowchuk NM, et al. Validity and reliability of wearable inertial sensors in healthy adult walking: a systematic review and meta-analysis. J Neuroeng Rehabil. 2020;17:62. https://doi.org/10.1186/s12984-020-00685-3.

11. Kirtley C, Whittle MW, Jefferson RJ. Influence of walking speed on gait parameters. J Biomed Eng. 1985;7:282-8. https://doi.org/10.1016/0141-542 5(85) $90055-X$

12. Ismailidis P, Egloff C, Hegglin L, Pagenstert G, Kernen R, Eckardt A, et al. Kinematic changes in patients with severe knee osteoarthritis are a result of reduced walking speed rather than disease severity. Gait Posture. 2020;79: 256-61. https://doi.org/10.1016/j.gaitpost.2020.05.008.

13. Andriacchi TP, Ogle JA, Galante JO. Walking speed as a basis for normal and abnormal gait measurements. J Biomech. 1977;10:261-8. https://doi.org/10.1 016/0021-9290(77)90049-5.

14. Bejek Z, Paróczai $R$, Illyés Á, Kiss RM. The influence of walking speed on gait parameters in healthy people and in patients with osteoarthritis. Knee Surg Sport Traumatol Arthrosc. 2006;14:612-22. https://doi.org/10.1007/s00167005-0005-6.

15. Peterson DS, Mancini M, Fino PC, Horak F, Smulders K. Speeding Up Gait in Parkinson's Disease. J Parkinsons Dis. 2020;10:245-53. https://doi.org/1 0.3233/JPD-191682.

16. van der Straaten R, De Baets $L$, Jonkers I, Timmermans A. Mobile assessment of the lower limb kinematics in healthy persons and in persons with degenerative knee disorders: A systematic review. Gait Posture. 2018;59:22941. https://doi.org/10.1016/j.gaitpost.2017.10.005

17. Leach JM, Mellone S, Palumbo P, Bandinelli S, Chiari L. Natural turn measures predict recurrent falls in community-dwelling older adults: a longitudinal cohort study. Sci Rep. 2018;8:4316. https://doi.org/10.1038/s41 598-018-22492-6.

18. Auvinet B, Touzard C, Montestruc F, Delafond A, Goeb V. Gait disorders in the elderly and dual task gait analysis: a new approach for identifying motor phenotypes. J Neuroeng Rehabil. 2017;14:7. https://doi.org/10.1186/ s12984-017-0218-1.

19. Mancini M, Schlueter H, El-Gohary M, Mattek N, Duncan C, Kaye J, et al. Continuous Monitoring of Turning Mobility and Its Association to Falls and Cognitive Function: A Pilot Study. Journals Gerontol Ser A Biol Sci. Med Sci. 2016;71:1102-8. https://doi.org/10.1093/gerona/glw019.

20. El-Gohary M, Pearson S, McNames J, Mancini M, Horak F, Mellone S, et al. Continuous Monitoring of Turning in Patients with Movement Disability. Sensors. 2013;14:356-69. https://doi.org/10.3390/s140100356.

21. Robinovitch SN, Feldman F, Yang Y, Schonnop R, Leung PM, Sarraf T, et al Video capture of the circumstances of falls in elderly people residing in long-term care: an observational study. Lancet. 2013;381:47-54. https://doi. org/10.1016/S0140-6736(12)61263-X

22. Woollacott M, Shumway-Cook A. Attention and the control of posture and gait: a review of an emerging area of research. Gait Posture. 2002;16:1-14. https://doi.org/10.1016/S0966-6362(01)00156-4

23. Abdallat $R$, Sharouf F, Button $K$, Al-Amri M. Dual-Task Effects on Performance of Gait and Balance in People with Knee Pain: A Systematic Scoping Review. J Clin Med. 2020;9:1554. https://doi.org/10.3390/jcm9051554.

24. lijima H, Equchi R, Aoyama T, Takahashi M. Trunk movement asymmetry associated with pain, disability, and quadriceps strength asymmetry in individuals with knee osteoarthritis: a cross-sectional study. Osteoarthr Cartil. 2019;27:248-56. https://doi.org/10.1016/j.joca.2018.10.012.

25. Reininga IHF, Stevens M, Wagenmakers R, Bulstra SK, Groothoff JW, Zijlstra W. Subjects with hip osteoarthritis show distinctive patterns of trunk movements during gait-a body-fixed-sensor based analysis. J Neuroeng Rehabil. 2012;9(3). https://doi.org/10.1186/1743-0003-9-3.

26. Bolink SAAN, Brunton LR, van Laarhoven S, Lipperts M, Heyligers IC, Blom AW, et al. Frontal Plane Pelvic Motion during Gait Captures Hip Osteoarthritis Related Disability. HIP Int. 2015;25:413-9. https://doi.org/10.53 01/hipint.5000282

27. Kellgren JH, Lawrence JS. Radiological Assessment of Osteo-Arthrosis. Ann Rheum Dis. 1957;16:494-502. https://doi.org/10.1136/ard.16.4.494.

28. de Groot IB, Favejee MM, Reijman M, Verhaar JAN, Terwee CB. The dutch version of the knee injury and osteoarthritis outcome score: A validation study. Health Qual Life Outcomes. 2008.

29. de Groot IB, Reijman M, Terwee CB, Bierma-Zeinstra SMA, Favejee M, Roos EM, et al. Validation of the Dutch version of the Hip disability and Osteoarthritis Outcome Score. Osteoarthr Cartil. 2007;15:104-9. https://doi. org/10.1016/j.joca.2006.06.014.

30. Morris R, Stuart S, McBarron G, Fino PC, Mancini M, Curtze C. Validity of Mobility Lab (version 2) for gait assessment in young adults, older adults and Parkinson's disease. Physiol Meas. 2019;40:095003. https://doi.org/10.1 088/1361-6579/ab4023.

31. Washabaugh EP, Kalyanaraman T, Adamczyk PG, Claflin ES, Krishnan C. Validity and repeatability of inertial measurement units for measuring gait parameters. Gait Posture. 2017;55:87-93. https://doi.org/10.1016/j.gaitpost.2 017.04.013.

32. Hoogkamer W, Bruijn SM, Duysens J. Stride length asymmetry in split-belt locomotion. Gait Posture. 2014;39:652-4. https://doi.org/10.1016/j.gaitpost.2 013.08.030.

33. Henderson EJ, Lord SR, Brodie MA, Gaunt DM, Lawrence AD, Close JCT, et al. Rivastigmine for gait stability in patients with Parkinson's disease (ReSPonD): a randomised, double-blind, placebo-controlled, phase 2 trial. Lancet Neurol. 2016;15:249-58. https://doi.org/10.1016/S1474-4422(15)003 89-0.

34. Dziuban CD, Shirkey EC. When is a correlation matrix appropriate for factor analysis? Some decision rules. Psychol Bull. 1974;81:358-61. https://doi.org/1 $0.1037 / \mathrm{h} 0036316$

35. Kaiser HF. The Application of Electronic Computers to Factor Analysis. Educ Psychol Meas. 1960;20:141-51. https://doi.org/10.1177/001316446002000116.

36. Gardner MJ, Altman DG. Confidence intervals rather than $P$ values: estimation rather than hypothesis testing. BMJ. 1986;292:746-50. https://doi. org/10.1136/bmj.292.6522.746

37. Ho J, Tumkaya T, Aryal S, Choi H, Claridge-Chang A. Moving beyond P values: data analysis with estimation graphics. Nat Methods. 2019;16:565-6. https://doi.org/10.1038/s41592-019-0470-3.

38. Verghese J, Robbins M, Holtzer R, Zimmerman M, Wang C, Xue X, et al. Gait Dysfunction in Mild Cognitive Impairment Syndromes. J Am Geriatr Soc 2008:56:1244-51. https://doi.org/10.1111/j.1532-5415.2008.01758.x.

39. Lord S, Galna B, Verghese J, Coleman S, Burn D, Rochester L. Independent domains of gait in older adults and associated motor and nonmotor attributes: Validation of a factor analysis approach. J Gerontol - Ser A Biol Sci Med Sci. 2013.

40. Horak FB, Mancini M, Carlson-Kuhta P, Nutt JG, Salarian A. Balance and Gait Represent Independent Domains of Mobility in Parkinson Disease. Phys Ther. 2016;96:1364-71. https://doi.org/10.2522/ptj.20150580.

41. Morris R, Hickey A, Del Din S, Godfrey A, Lord S, Rochester L. A model of free-living gait: A factor analysis in Parkinson's disease. Gait Posture. 2017;52: 68-71. https://doi.org/10.1016/j.gaitpost.2016.11.024.

42. Arcolin I, Corna S, Giardini M, Giordano A, Nardone A, Godi M. Proposal of a new conceptual gait model for patients with Parkinson's disease based on factor analysis. Biomed Eng Online. 2019:18-70. https://doi.org/10.1186/s12 938-019-0689-3.

43. Astephen Wilson JL. Challenges in dealing with walking speed in knee osteoarthritis gait analyses. Clin Biomech. 2012.

44. Senden R, Grimm B, Meijer K, Savelberg H, Heyligers IC. The importance to including objective functional outcomes in the clinical follow up of total knee arthroplasty patients. Knee. 2011;18:306-11. https://doi.org/10.1016/j. knee.2010.07.008.

45. Reininga IHF, Stevens M, Wagenmakers R, Boerboom AL, Groothoff JW, Bulstra SK, et al. Compensatory Trunk Movements in Patients with Hip 
Osteoarthritis. Am J Phys Med Rehabil. 2011;90:681-7. https://doi.org/10.1 097/PHM.0b013e31820f955e.

46. Watelain E, Dujardin F, Babier F, Dubois D, Allard P. Pelvic and lower limb compensatory actions of subjects in an early stage of hip osteoarthritis. Arch Phys Med Rehabil. 2001;82:1705-11. https://doi.org/10.1053/apmr.2 001.26812.

47. Zeni J, Pozzi F, Abujaber S, Miller L. Relationship between physical impairments and movement patterns during gait in patients with end-stage hip osteoarthritis. J Orthop Res. 2015;33:382-9. https://doi.org/10.1002/jor.22 772.

48. de Reus IMA, Spekenbrink-Spooren A, van Steenbergen LN, Denissen GAW, Rijnsburger E, van der Togt CR. LROI Annual Report 2019. 's-Hertogenbosch; 2019.

\section{Publisher's Note}

Springer Nature remains neutral with regard to jurisdictional claims in published maps and institutional affiliations.

Ready to submit your research? Choose BMC and benefit from:

- fast, convenient online submission

- thorough peer review by experienced researchers in your field

- rapid publication on acceptance

- support for research data, including large and complex data types

- gold Open Access which fosters wider collaboration and increased citations

- maximum visibility for your research: over $100 \mathrm{M}$ website views per year

At BMC, research is always in progress.

Learn more biomedcentral.com/submissions 